\title{
Congenital Absence of Meibomian Glands: report of two cases
}

\author{
Rahimi F. ${ }^{1}$, Abdi F. ${ }^{1}$, Mafi M. ${ }^{1}$,Esfahani M. ${ }^{2}$, Faramarzi N. ${ }^{1}$ \\ ${ }^{\prime}$ Farabi Eye Hospital, Tehran University of Medical Sciences, Tehran, Iran' \\ 2Department of Ophthalmology, Faculty of Medicine, Ahwaz Jondi Shapour \\ University of Medical Sciences, Ahwaz, Iran.
}

\begin{abstract}
A 36-year-old man with his 11-year-old son presented with ocular surface irritation symptoms and blurred vision since early childhood, on exam visual acuity of the man was $5 / 10(O D), 4 / 10(O S)$ and his son was 6/10(OD), 5/10(OS), other exams of these patients were similar: eyelid examination showed absence of meibomian gland orifices, after application of fluorescein dye there was a thin tear film layer and diffuse punctuate epithelial erosions on the cornea, remaining exams were unremarkable.
\end{abstract}

Key words: absent meibomian gland, ectodermal dysplasia, dry eye, congenital meibomian gland anomalies, congenital absence of meibomian gland

\section{Introduction}

The meibomian glands play a key role in maintaining a healthy ocular surface by secreting lipids that help to stabilize the tear film, the meibomian glands minimize the evaporative loss of tear fluid. Maintaining aqaqueous tear volume keeps the ocular surface protected throughout the blink cycle and reduces the risk of hyperosmolaritiy ,meibomian glands abnormalities may create significant ocular surface problems, when patients don't have enough of the meibomian gland-secreted lipids in tear film to prevent evaporation, they can develop dry eye, even if aqueous production remains normal.

\section{Case presentation}

A 36-year -old with his 11-year-old son were referred to farabi eye hospital cornea clinic complaining of ocular surface irritation symptoms like foreign body sensation , tearing and blurred vision since early childhood, the man mentioned similar symptoms in his father. On presentation visual acuity of the man was 5/10(OD), 4/10(OS) and his son was 6/10(OD), 5/10(OS), other exams were similar in both patients: external exams including eyelid and adnexa showed absence of meibomian orifices both in the upper and lower eyelids, conjunctiva was mildly injected, the corneal surface was irregular and staining with fluorescein showed diffuse punctuate epithelial erosions and a very thin tear film layer (figures 1-4).

The anterior chamber, lens and fundus were unremarkable.Their past medical history was negative and they didn't have any systemic complaints except for difficulty in swallowing.

Correspondence: Mostafa Mafi, Farabi Eye Hospital, Tehran University of Medical Science, Qazvin Sq., Tehran 13336, Iran

E-mail: mostafa.mafi17@gmail.com 
Rahimi, Abdi, Mafi, et al
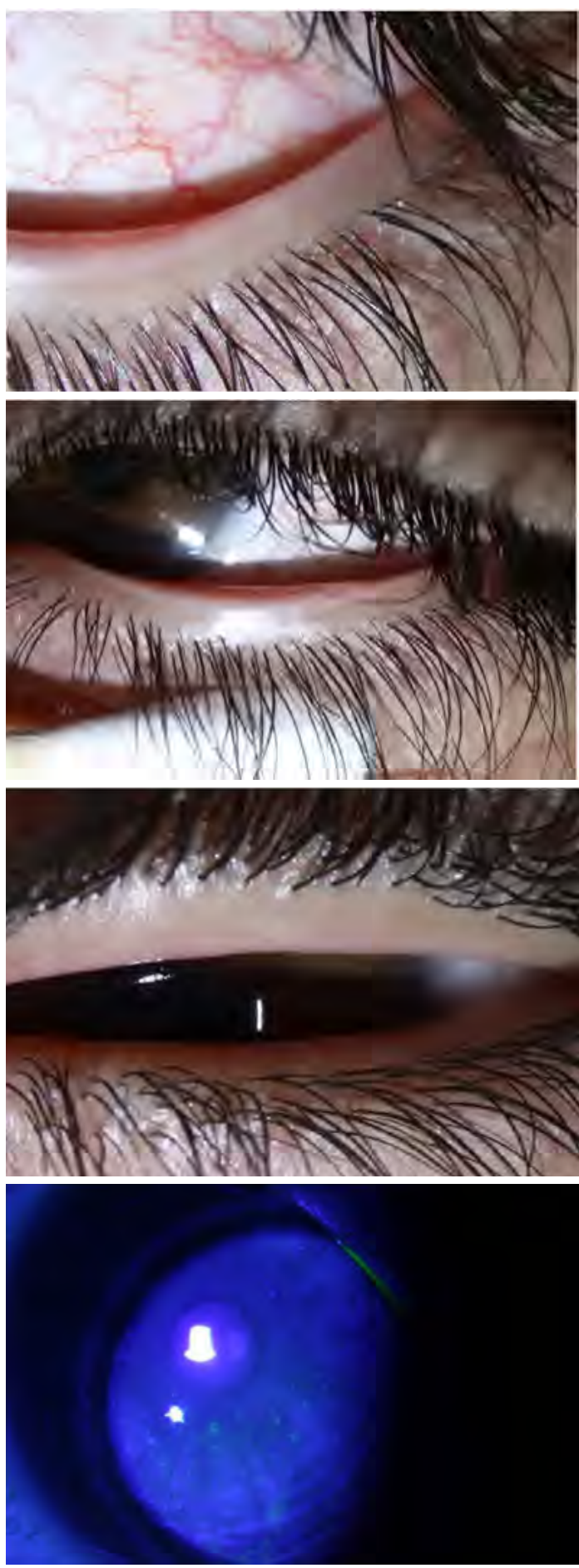

Fig.1: Absence of meibomianglands and orifices in the lowereyelid of the Son

Fig. 2: Absence of meibomian orifices in the lower eyelid of the Son

Fig.3: Absence of meibomian glands and orifices in the upper eyelid of the Father

Fig 4: Irregular corneal surface stained with fluorescein showed diffuse punctuate epithelial erosions and a very thin tear film layer 


\section{Discussion}

The ectodermal dysplasia syndromes are congenital disorders that are identified by the absence or deficient function of at least two derivatives of the ectoderm such as teeth, hair, nails and sweat glands ${ }^{1}$.

Meibomian gland deformities and tear film disorders have been described in patients with ectodermal dysplasia syndrome. ${ }^{2-4}$ Congenital absence of meibomian glands is exceedingly rare, ${ }^{3,5}$ but has been reported in ectodermal dysplasiasyndrome and ill anhidrotic ectodermal dysplasia. ${ }^{2-6}$

T. Kaercher studied thirty-six patients with ectodermal dysplasia syndromes. Transillumination of the meibomian glands was performed on 22 of them and revealed alterations of the meibomian glands in 21 patients (95.45\%). These alterations included partial loss of the glands, coarsening of the acini and complete absence of meibomian glands. ${ }^{7}$ Mondino ${ }^{8}$ histologically showed an absence of meibomian orifices at the upper and lower lid.

The biomicroscopic absence of meibomian orifices was reported by Koniszewski ${ }^{9}$, but not confirmed by histologic series. Full -thickness biopsies cannot be performed routinely for clinical purposes. The technique of transillumination (meibomianoscopy) provides useful information, and in contrast to histologic techniques, all areas of the upper and lower lid can be inspected. The meibomianoscopy provides information about regularity, differentiation, size and number of the meibomian glands. Therefore, we chose this technique for our case report. In our cases, there were no meibomian glands based on biomicroscopic study, (Fig 1-2) but there was no histopathologic study. Our patients showed no other sign of ectodermal dysplasia syndromes except for complete absence of meibomian glands in both lids of both eyes.

In the study by Bron and Tripathi, a particular form of epithelial surface change was observed, which was associated with the meibomian blockade. ${ }^{5}$ In that situationepithelial cysts are observed in the exposed region of the cornea, together with punctate erosions and punctate epithelial keratopathy. In our patients, due to lack of normal meibomian glands secresions, conjunctival injection, altered tear film and punctuate epithelial erosions were seen (Fig 3). Our cases showed absence of meibomian glands in both lids of both eyes, but they were otherwise healthy with difficulty in swelling.

Preservative-free artificial tears (Artelac ${ }^{\oplus}$, Bausch and Lomb Company, Rochester, NY) was prescribed every 6 hours as well as eye gel (Liposic ${ }^{\circledR}$ Ophtalmic Liquid Gel) before sleep for both patients. 


\section{References}

1. Brenner S, Miller JH (2001) Encyclopedia of genetics. Academic Press, San Diego, pp 559-601

2. Elmann, S., Hanson, S. A., Bunce, C. N., \& Shinder, R. (2014). Ectrodactyly Ectodermal Dysplasia Clefting (EEC) Syndrome: A Rare Cause of Congenital Lacrimal Anomalies. Ophthalmic plastic and reconstructive surgery.

3. Allali, J., Roche, O., Monnet, D., Brezin, A., Renard, G., \& Dufier, J. L. (2007). [Anhidrotic ectodermal dysplasia:" congenital ameibomia"]. Journal francais d'ophtalmologie, 30(5), 525-528.

4. Ireland IA, Meyer DR (1998) Ophthalmic manifestations of ectrodactyly-ectodermal dysplasiaclefting syndrome. Ophthalmic PlastReconstrSurg 14:295-297

5. Bron AJ, Benjamin L, Snibson GR. Meibomian gland disease: classification and grading of lid changes. Eye 199 1; 5:39 5-411.

6. Ekins $\mathrm{MBC}$, Waring GO. Absent meibomian glands and reduced corneal sensation in hypo hidrotic ectodermal dysplasia. J PediatrOphthalmol Strabismus 1981; 18: 44-7.

7. T. Kaercher, Ocular symptoms and signs in patients with ectodermal dysplasia syndromes, Graefe's Arch ClinExpOphthalmol (2004) 242:495-500.

8. Mondino BJ, Bath PE, Foos RY et al (1984) Absentmeibomian glands in the ectrodactyly, ectodermal dysplasia, cleft lip-palate syndrome. Am J Ophthalmol 97:496-500

9. Koniszewski G, Maywald M, Henke V (1987) AugenbeteiligungbeiektodermalerDysplasie. KlinMonatsblAugenheilkd 190:519-523 